\title{
Research on diagram automatic generation based panorama dispatching technologies for smart grid
}

\author{
Wenchong Fang ${ }^{1}$,Taifeng $\mathrm{Kang}^{2,}{ }^{\text {a }}$, Yonggang $\mathrm{Li}^{2}$, Shouyu Liang ${ }^{1}$, Ruipeng \\ Zhang $^{1}$, Fengqing Zhao ${ }^{1}$, Bin Zhang ${ }^{2}$ \\ ${ }^{1}$ China Southern Power Grid (CSG) dispatching and control center, Guangzhou, China \\ ${ }^{2}$ Beijing Sifang Automation CO., LTD., Beijing, China \\ aarfa2011_1@163.com \\ * Corresponding author
}

Keywords: power flow diagram; panorama dispatching; diagram automatic generation; geographic information; smart grid

\begin{abstract}
In modern power grid, especially with the development of ultra high voltage (UHV) and AC-DC hybrid power grid, the scale and complexity of power transmission network increase a lot. System operators need to quickly grasp real-time operation situation and make decision to ensure the security and economics of the grid. GUI (graphical user interfaces) has become essential components of modern control center. Power flow diagrams and other diagrams such as substation diagrams present the real-time situation and provide the interface for remote control. Manually creation and maintenance of such diagrams is totally unacceptable as it is very time consuming and error prone. In this paper, we proposed a panorama dispatching framework, which is based on diagram automatic generation technologies to reveal different levels of details of the power grid and realize system situation awareness and decision support. Panorama dispatching and system situation aware applications have been developed based on our proposed framework in China Southern Power Grid (CSG) dispatching and control center. Actual examples are provided to prove the feasibility and advantage of our proposed framework. The work in this paper is very useful for increasing the efficiency for model and diagrams management, system operation and situation awareness.
\end{abstract}

\section{Introduction}

In modern power grid, especially with the development of ultra high voltage (UHV) and AC-DC hybrid power grid, the scale and complexity of power transmission network increase a lot. System operators need to quickly grasp real-time operation situation and make decision to ensure the security of the grid [1]. As the toolkits for interacting with system operators, GUI (graphical user interfaces) has become essential components of modern control center [2]. Power flow diagrams and other diagrams such as substation diagrams present the real-time situation and provide the interface for remote control. In traditional EMS (Energy Management System), such diagrams are manually generated and maintained. In the new era of smart grid, manually creation and maintenance of such diagrams is totally unacceptable as it is very time consuming and error prone. As one of the emerging technologies of smart grid, diagram automatic generation has draws more and more attentions [3, 4, 5].

In this paper, we proposed a panorama dispatching framework, which is based on diagram automatic generation technologies to reveal different levels of details of the power grid and realize system situation awareness and decision support. The framework is shown in Fig.1. The framework could provide system operators with panorama vision of power grid, and increase the efficiency for model and diagrams maintenance and system operation. 


\section{Diagram automatic generation for smart grid}

Smart grid. In traditional power grid, there is very little flexibility, limited self-healing ability and information sharing and utilization for operation of power supply and power transmission.

Smart grid is based on strong and reliable, flexible and interactive platform to optimize security, efficiency, economics and sustainability of the grid by utilizing the panoramic information. In the control center of smart grid, comprehensive and decision supporting information is available for system planning, operation schedule and real-time operation. All kinds of tools are developed to provide analysis, diagnosis and optimization for system operation.

Diagram automatic generation. Diagram automatic generation is based on auto-routing technology, to automatically layout the equipments on the diagram to effectively organize the elements on the diagram and provide information sharing and utilization. Diagram automatic generation can increase the efficiency for model and diagrams management and system operation. Generally, there're three types of diagrams associated with system management and operation, including single-line substation diagram, control area based diagram and geographic information based power flow diagram.

Single-line diagram. This type of diagram provides the layout of the equipments in the substation, including bus bar, transformer, circuit breaker, load, etc. It is the basic diagram for system operators to invest on system operation situation and perform remote control. However, information is limited to single substation and operators need to jump among multiple diagrams when necessary, which is very time consuming and inconvenient.

Control area based power flow diagram. This type of diagram provides higher level of details than the single-line diagram with the interconnection information between different control areas. It can provide systematic view of the control areas and it is suitable for system operation and dispatch.

Geographic information based power flow diagram. This type of diagram provides geographic and systematic view of the total transmission network. It is suitable to provide system wide situation awareness and decision support.

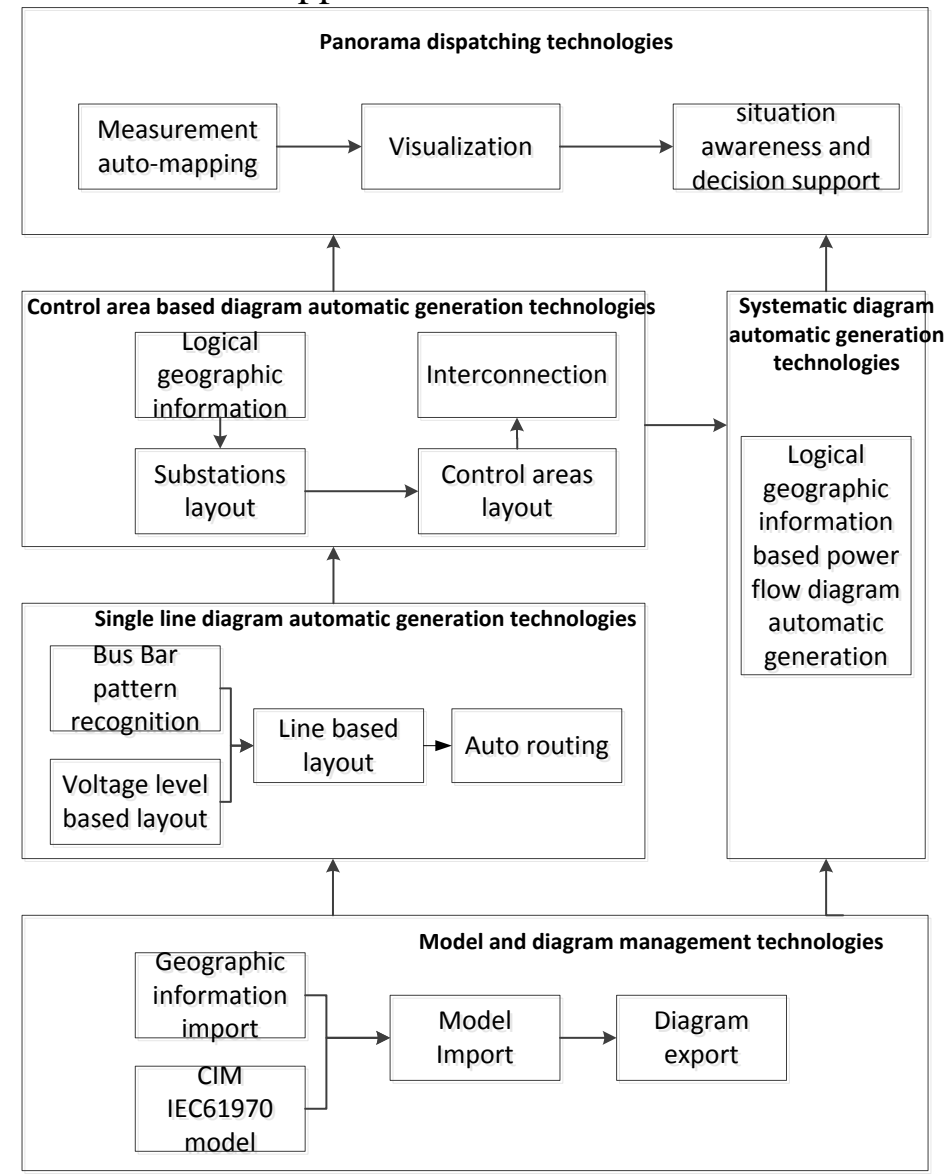

Fig. 1 The Panorama dispatching technology framework 


\section{Key technologies for diagram automatic generation based panorama dispatching}

Model and diagram management technologies. Model and diagram management technologies are the fundamental for panorama dispatching technologies.

Geographic information import. Geographic information is important for systematic level diagram generation to provide geographical perspective of the system, fast location of specific area and substation and auxiliary decision support.

IEC61970 CIM based model export. IEC61970 CIM is the information modeling and exchange standard. Import and Export model based on IEC61970 CIM provides the ability of model consistence examination with the CIM standard as well as formation modeling and exchange between systems and applications.

Diagram export. The automatically generated diagrams are of high level abstraction and reorganization of model and information; there is the opportunity to integrate the diagrams with other advanced application in EMS. With Diagram export technology, all the diagrams could be exported into EMS and other existing system, enhancing integration and utilization level of both panorama diagrams and existing system.

Single line diagram automatic generation. Based on the model of distribution system, generate the details in substation levels to provide the basic view of system operation and interfaces for remote control..

Voltage level based layout. The single line diagram is generally composed of several parts according to the voltage levels in the substations. Voltage level is the criterion for dividing the diagram in to different regions. Voltage level based layout technology organizes the layout according to the voltage level in the substations and keeps the generated diagram legible and well-organized.

Bus bar pattern recognition. Bus Bar is the essential part in the layout of the equipments in different voltage levels. There are different patterns of bus connection such as parallel bus, splitting bus and 3/2 bus connection, etc. Incorrect recognition of the pattern may affect the layout of entire diagram. Bus bar pattern recognition is one of the key technologies for single line diagram automatic generation.

Row based layout. The layout of single line diagram is in straight matrix. The equipments such as bus bars, transformers and so on are organized on the diagram in different rows and columns. Row based layout simplifies the procedure for single line diagram generation and keeps the diagram clear and elegant.

Auto routing. Auto routing technologies are essential for panorama diagrams generation which automatically find route and connect the equipments in the substation to generate interconnected diagram. There are common requirements for auto routing technologies: no overlap, minimum crossings and shortest path, etc.

Control area diagram automatic generation. Abstract the details inside the substations and disclosure of the details of interconnection between substations and control areas, to provide systematic view of power transmission network.

Logical geographic information. Geographic information is important for systematic perspective of the transmission network. However, it is not applicable to utilize geographic information for diagram generation directly. Logical geographic information is based on the original geographic information to balance the layout of the diagram, prevent the crowd and disorder on the diagram and increase the clarity and elegancy of the generated diagram.

Substation layout. Abstract the details inside the substation and reserve the substation in the layout according to the geographic information in the specific control area it belongs to. The layout of substations needs automatically adjustment so as to keep clarity and elegancy.

Control areas layout. With the entire substations in the control areas, different control areas need to layout according to geographic information and keep clarity and elegancy, too.

Interconnection. According to the sketch of the transmission lines, the substations need to be interconnected across the direction that connects the centers of the different substations. Besides, 
there may be T-connect lines, and the interconnection of lines needs to ensure minimum length of lines and no or less crossings.

Systematic diagram automatic generation. Systematic diagram utilizes and reorganize the separate information scattered in different substations and generate the panorama diagrams for system operators to provide systematic vision of the entire power transmission network.

Logical geographic information based power flow diagram automatic generation. This technology utilizes the logical geographic information of substations in different control areas to generate systematic interconnected power flow diagram. The generated diagram provides panorama vision of the power transmission network with geographic information for reference and it is applicable for situation awareness and decision support applications.

Panorama dispatching technologies. Panorama diagrams provide multiple visions of power transmission network by integration with advanced system analysis and operation technologies. The generated panorama diagrams can be developed as a unique strategic diagram to satisfy the most operation and status perception requirements for system operators and provide advanced efficiency and convenience.

Measurement auto-mapping. Measurements are basic data and information for system operation to percept the system operation situation and perform advanced analysis and control. Automatically measurement mapping is quite necessary to provide fundamental information for advanced panorama dispatching and control technologies and applications.

Visualization. The measurements are raw and difficult to be recognized by system operators. Visualization technologies are based on computer graphic and information reorganizing methods to reveal the information behind the digital number of measurements in visual and vivid way. Visualization is key technology for smart grid.

Situation awareness and decision support. Based on the measurements, multiple dispatching applications can be applied to achieve better perception and prediction on the system operation situation. According to these situation awareness results, system operators could quickly grasp the real-time system operation situation, potential risks that may decrease the security, economics or efficiency level and provide possible operation strategies to eliminate or reduce relative impact. Situation awareness and decision support technologies based on panorama diagrams to provide higher visions of the whole distribution system and smart dispatching applications.

\section{Case study}

Based on the proposed technology framework, we have built up system panorama diagram automatic generation based situation awareness and decision support system, which automatically generate single-line, control area based and logical geographic information based panorama diagrams for panorama dispatching and decision support. Fig. 2 shows the control area based panorama diagram (a) and logical geographic information based panorama diagram of different regions of power transmission network. From this diagram, we can easily get the landscape of the power transmission network and its real-time operation status, and utilize these diagrams to guide system dispatch and optimization from panorama perspective.

\section{Conclusions}

In this paper, we proposed a panorama dispatching framework, which is based on diagram automatic generation and different information abstraction and reorganization of substation and geographic information for power transmission network. Based on the framework, panorama visions of transmission network of large scale power grid are provided to the system operators as well as the advantages of situation awareness and decision support. The proposed technology framework has been applied in actual power transmission system and real examples have shown its advantages of our diagram automatic generation based panorama diagram panorama dispatching and control for smart grid. 


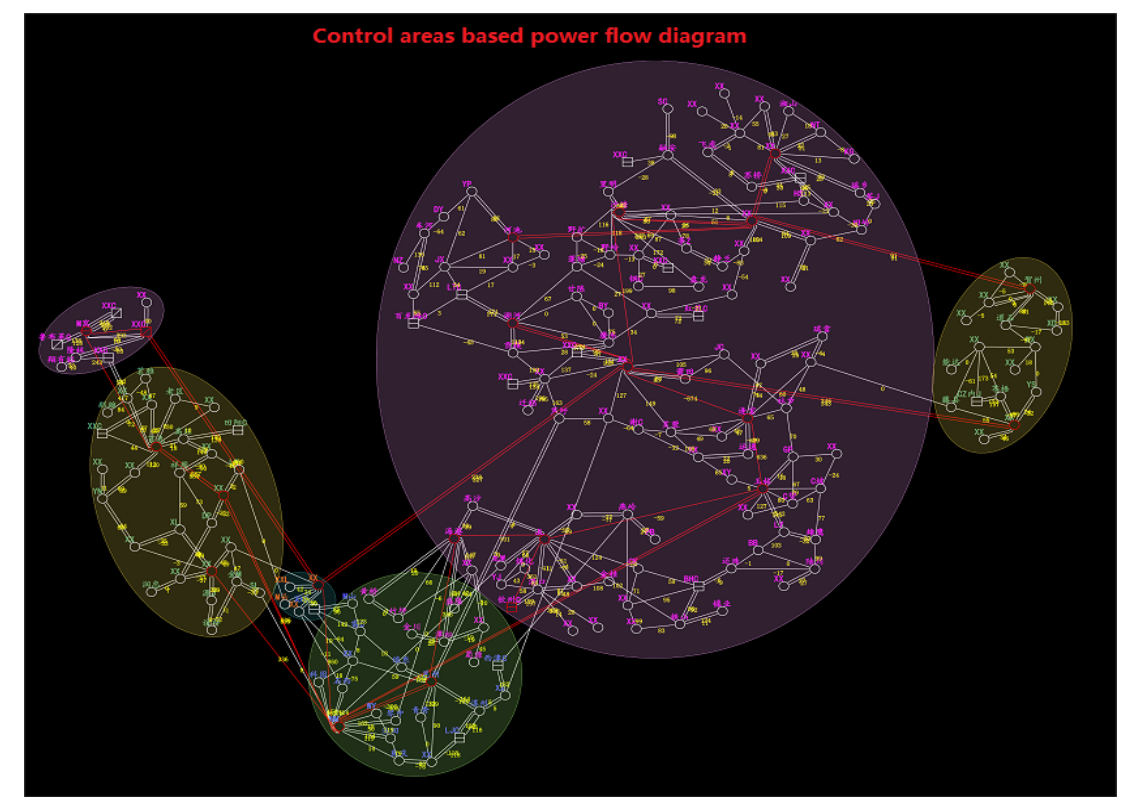

(a) Control area based power flow diagram

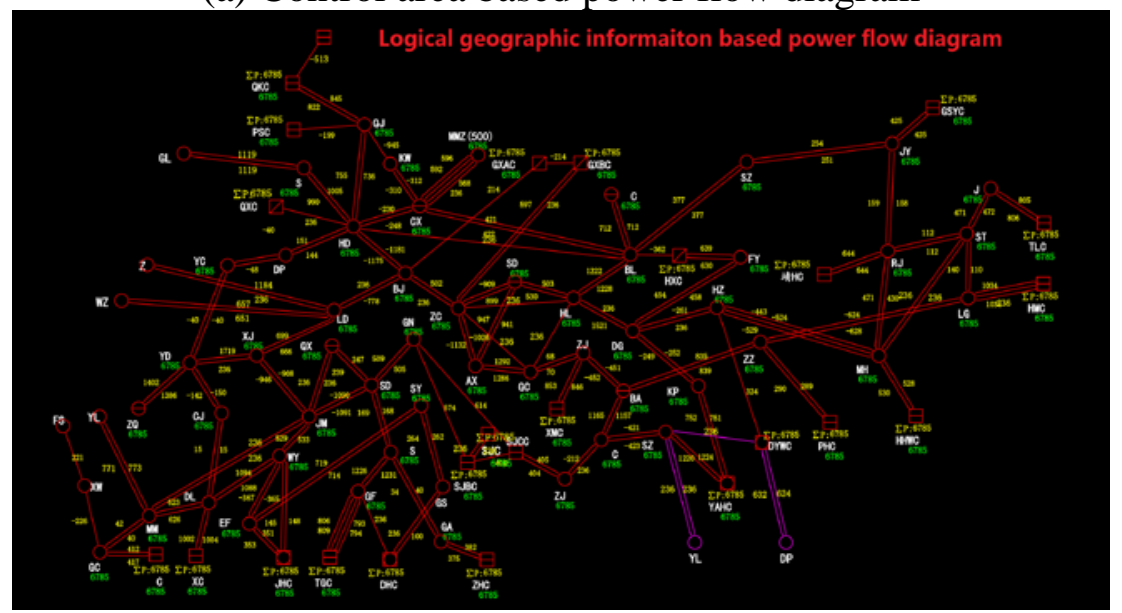

(b) Logical geographic information based power flow diagram

Fig. 2 Examples of panorama diagram in actual panorama dispatching project

\section{Acknowledgments}

This work is sponsored by China Southern Power Grid (CSG) Technology Program (No. K-ZD2013-006.)

\section{References}

[1] AMIN S M, GIACOMONI A M: IEEE Power and Energy Magazine, Vol. 10(2012) 33-40.

[2] Rochefort, M, De Guise, N, Gingras, L: Digital Power System Simulators, (1995) 129.

[3] Ong, Y.S, Gooi, H.B, Chan, C.K: Generation, Transmission and Distribution, IEE Proceedings, Vol.147(2000) 292-298.

[4] ZHANG Jianmin, FANG Wendao, HU Bing, XU Guanhua: Automation of Electric Power Systems, Vol.37(2012) 72-76

[5] ZHANG Jianmin, YE Yi, XU Guanhua: Automation of Electric Power Systems, Vol.37(2013) 84-91. 